\title{
Drug Content Uniformity: Quantifying Loratadine in Tablets Using a Created Raman Excipient Spectrum
}

\author{
Amelia Farquharson ${ }^{1}$, Zachery Gladding ${ }^{2}$, Gary Ritchie ${ }^{3}$, Chetan Shende ${ }^{2}$, Joseph Cosgrove ${ }^{4}$, Wayne Smith ${ }^{5}$, \\ Carl Brouillette ${ }^{2}$ and Stuart Farquharson ${ }^{2, *}$
}

1 Rennselaer Polytechnic Institute, Troy, NY 12180, USA; amy.farquharson@yahoo.com

2 Real-Time Analyzers, Inc., Middletown, CT 06457, USA; zachary.gladding@gmail.com (Z.G.); chetan@rta.biz (C.S.); carl@rta.biz (C.B.)

3 GER Compliance, Fenton, MO 63026, USA; gary.e.ritchie@gmail.com

4 Advanced Fuel Research, Inc., East Hartford, CT 06108, USA; cosgrove@afrinc.com

5 Northrup Grumman, East Hartford, CT 06108, USA; wayne@wsmithconsulting.com

* Correspondence: stu@rta.biz; Tel.: +860-635-9800

Citation: Farquharson, A.; Gladding, Z.; Ritchie, G.; Shende, C.; Cosgrove, J.; Smith, W.; Brouillette, C.;

Farquharson, S. Drug Content

Uniformity: Quantifying Loratadine in Tablets Using a Created Raman Excipient Spectrum. Pharmaceutics 2021, 13, 309. https://doi.org/ 10.3390/pharmaceutics13030309

Academic Editor: Juan Torrado

Received: 22 January 2021

Accepted: 21 February 2021

Published: 27 February 2021

Publisher's Note: MDPI stays neutral with regard to jurisdictional claims in published maps and institutional affiliations.

Copyright: () 2021 by the authors. Licensee MDPI, Basel, Switzerland. This article is an open access article distributed under the terms and conditions of the Creative Commons Attribution (CC BY) license (https:// creativecommons.org/licenses/by/ $4.0 /)$.

\begin{abstract}
Raman spectroscopy has proven valuable for determining the composition of manufactured drug products, as well as identifying counterfeit drugs. Here we present a simple method to determine the active pharmaceutical ingredient (API) mass percent in a sample that does not require knowledge of the identities or relative mass percents of the inactive pharmaceutical ingredients (excipients). And further, we demonstrated the ability of the method to pass or fail a manufactured drug product batch based on a calculated acceptance value in accordance with the US Pharmacopeia method for content uniformity. The method was developed by fitting the Raman spectra of 30 Claritin ${ }^{\circledR}$ tablets with weighted percentages of the Raman spectrum of its API, loratadine, and a composite spectrum of the known excipients. The mean loratadine mass of $9.79 \pm 40 \mathrm{mg}$ per $100 \mathrm{mg}$ tablet compared favorably to the $10.21 \pm 0.63 \mathrm{mg}$ per $100 \mathrm{mg}$ tablet determined using highperformance liquid chromatography, both of which met the acceptance value to pass the $10 \mathrm{mg}$ API product as labelled. The method was then applied to a generic version of the Claritin product that employed different excipients of unknown mass percents. A Raman spectrum representative of all excipients was created by subtracting the API Raman spectrum from the product spectrum. The Raman spectra of the 30 generic tablets were then fit with weighted percents of the pure loratadine spectrum and the created excipient spectrum, and used to determine a mean API mass for the tablets of $10.12 \pm 40 \mathrm{mg}$, again meeting the acceptance value for the $10 \mathrm{mg}$ API product. The data suggest that this simple method could be used to pass or fail manufactured drug product batches in accordance with the US Pharmacopeia method for content uniformity, without knowledge of the excipients.
\end{abstract}

Keywords: active pharmaceutical ingredient; drug content uniformity; acceptance value; process control; Raman spectroscopy; loratadine

\section{Introduction}

In the past two decades, Raman spectroscopy has become an important analytical tool for determining the amount of a pharmaceutical active ingredient (API) in solid dose medications [1-8]. Knowledge of the API mass, or mass percentage, is essential in the development and quality control of drug product formulations, as well as in the identification of counterfeit drug products [9-11]. The fact that the API mass percent in tablet medications can be determined non-destructively, without sample preparation, and within a few minutes makes Raman spectroscopy ideal for these applications. Consequently, Raman spectroscopy is being used in pharmaceutical company laboratories and production facilities, as well as by enforcement agencies, such as the US Food and Drug Administration [9]. However, making quantitative measurements is challenging. The problem is 
two-fold. First, the uniformity of the API in a tablet decreases with its mass percent, and second, the size of the focal volume of a typical laser used to generate Raman radiation is several orders of magnitude smaller than the volume of a tablet. Consequently, a single point measurement could miss the API, especially in the case of low dose tablets.

There are a number of publications describing various optical schemes to overcome this under-sampling of the API in such drug products [12-17]. The first approach is to simply map the entire surface volume of a tablet, either point-by-point, line scanning, or imaging using back-scattering geometry $[6,13,14]$. While these approaches generally only measured the surface volume to a depth of 1-2 $\mathrm{mm}$, good quantitative results can be obtained. However, these mapping approaches often take an hour or more. More recently, forward scattering Raman [18], now frequently called transmission Raman [16,17,19], has been developed to measure most of the tablet by collecting the Raman radiation on the tablet side opposite the laser irradiation. While this has proven successful in some cases, it suffers from a decrease in Raman signal intensity by at least an order of magnitude; and, more importantly, scattering due to inactive pharmaceutical ingredients (or excipients), such as diluents and binders, can lead to predicted API mass percents that are incorrect by more than a factor of two [19].

However, in virtually all of these publications, the approach is tailored to a single medication, where model tablets are prepared to mimic products, so that mass percentage plots can be prepared to quantify the API in actual products [7]. This approach may work when the API represents the major portion of the tablet, or only 1 or 2 excipients are present, and known. However, in most tablets, there are at least 4 excipients and sometimes more than 10. Furthermore, the relative excipient mass percentages are often a trade secret, making analysis particularly difficult for forensic samples [20].

Here, we describe a method to overcome this limitation imposed by the excipients by creating a composite Raman spectrum of all of the excipients, which can then be used to quantify the API mass percentage with high accuracy and precision. The method was applied to the determination of the mass percentage of loratadine, the API in the original product Claritin ${ }^{\circledR}$ and the mass percentage of loratadine in an alternative generic product. In addition, the data were compared to high-performance liquid chromatography data, and both data sets were evaluated in terms of acceptance values for passing or failing a manufactured lot, as defined by the US Pharmacopeia (USP) procedure to test dosage content uniformity [21]. Claritin was chosen for this study to represent moderately low dose drugs that require measuring the API mass percent for such USP tests, and not simply the total tablet mass, as is the case for high dose drugs.

\section{Materials and Methods}

\subsection{Materials}

Loratadine, desloratadine, lactose monohydrate, corn starch and magnesium stearate were purchased from USP (Rockville, MD, USA), while acetonitrile, de-ionized (DI) water, phosphoric acid, and potassium dihydrate were purchased from Sigma Aldrich (St. Louis, $\mathrm{MO}$, USA). Reference tablets at $1 \mathrm{~cm}$ diameter and $\sim 400 \mathrm{mg}$, composed of the USP grade Claritin chemical components, loratadine, magnesium stearate, lactose monohydrate and cornstarch, were prepared using a hydraulic press set to 1500 psi. Tablets of Claritin and a generic product (Schering-Plough, Memphis, TN, USA and MSD Consumer Care, Whitehouse Station, NJ, USA, respectively) were purchased from a local pharmacy. Thirty of the Claritin tablets were separately weighed prior to Raman and HPLC measurements.

\subsection{Method 1: Raman Spectroscopy}

All Raman spectroscopic measurements were performed using a LabRaman Analyzer (Real-Time Analyzers, Inc., RTA, Middletown, CT, USA). The analyzer employed a $1064 \mathrm{~nm}$ laser that provided a $275 \mathrm{~mW}, \sim 100 \mu \mathrm{m}$ diameter spot, and a diffraction grating-InGaAs array detector combination that provided a 200 to $1800 \mathrm{~cm}^{-1}$ spectral region with $\sim 10 \mathrm{~cm}^{-1}$ resolution. The tablets were placed in a machined plate, designed to hold various size and 
shape tablets, and mounted above the laser on an XY positioning stage (Conix Research, Springfield, OR, USA). The stage positioning resolution is $\sim 1 \mu \mathrm{m}$, with an XY repeatability of $\sim 20 \mu \mathrm{m}$. A $9 \times 9$ grid covering a $4 \times 4 \mathrm{~mm}^{2}$ section of one Claritin tablet having a diameter of $6.35 \mathrm{~mm}$ and a thickness of $2.44 \mathrm{~mm}$ was measured using the XY stage. The laser focal point was set to a depth of $\sim 1 \mathrm{~mm}$ below the surface and scanned back and forth over a distance of $3.3 \mathrm{~mm}$ for each of the 81 spectra, which consisted of 10 averaged, 4-s integrations (total time at $40 \mathrm{~s} / \mathrm{spot}$ was $\sim 1 \mathrm{~h}$ ) [12].

Heat maps, indicating the loratadine mass percent, were prepared for this Claritin tablet using Plotly Chart Studio software (Montreal, Canada). The loratadine mass percentage for this tablet was also determined using S-Quant software (version 1.3.5 RTA) [22]. This and the remaining tablets were each measured as a $3 \times 3$ grid covering $2 \times 2 \mathrm{~mm}^{2}$ to a depth of $\sim 1 \mathrm{~mm}$. The 9 spectra, consisting of 10 averaged, 2-s integrations ( $3 \mathrm{~min} / \mathrm{tablet}$ ), were averaged and then treated using a 3rd-order, 13-point running smooth. The S-Quant software was used to determine the mass percents for all remaining tablets.

\subsection{Method 2: High-Performance Liquid Chromatography}

The HPLC analysis was performed using a Shimadzu LC-10ATvp (Kyoto, Japan) with a $\mathrm{D}_{2}$ lamp and a $254 \mathrm{~nm}$ detector. A Supelco C8 $5 \mu \mathrm{m} 15 \mathrm{~cm} \times 4.6 \mathrm{~mm}$ column (Center County, PA, USA) was selected to satisfy the USP L1 phase requirement. A buffer solution was prepared by dissolving $1.75 \mathrm{~g}$ potassium dihydrate in $250 \mathrm{~mL}$ DI water, which was adjusted to a $\mathrm{pH}$ of 3.5 using phosphoric acid [23]. A mobile phase was prepared by adding $150 \mathrm{~mL}$ of buffer to $350 \mathrm{~mL}$ of acetonitrile, which was sonicated for $20 \mathrm{~min}$ and then passed through a $0.45 \mu \mathrm{m}$ filter. An internal standard was prepared by adding $400 \mathrm{mg}$ of USP desloratadine to $400 \mathrm{~mL}$ of the mobile phase, while a calibration series for loratadine was prepared at $0.05,0.10,0.15,0.20$, and $0.25 \mathrm{mg} / \mathrm{mL}$ in the mobile phase by diluting a $1 \mathrm{mg} / \mathrm{mL}$ loratadine reference standard (Sigma Aldrich). Each concentration was measured three times, and the averages were used to prepare the calibration plot. Each Claritin tablet, previously measured by Raman, was weighed, then crushed by mortar and pestle, placed in a $20 \mathrm{~mL}$ glass vial, to which $10 \mathrm{~mL}$ of the internal standard mobile phase was added. Each sample was vortexed and sonicated to dissolve the tablet. These samples were further dilution by adding $1 \mathrm{~mL}$ of the dissolved sample into $9 \mathrm{~mL}$ of the internal standard mobile phase to yield an approximate $0.1 \mathrm{mg} / \mathrm{mL}$ concentration for both desloratadine and loratadine. These samples were also sonicated for $20 \mathrm{~min}$ and passed through $0.45 \mu \mathrm{m}$ filters.

The column was heated to $35^{\circ} \mathrm{C}$ and conditioned first using a 50:50 v/v acetonitrile/DI water solution for $30 \mathrm{~min}$, and second with the mobile phase, which was flowed at $0.5 \mathrm{~mL} / \mathrm{min}$ for $15 \mathrm{~min}$, then $1.0 \mathrm{~mL} / \mathrm{min}$ for an additional $15 \mathrm{~min}$. For each sample, $20 \mu \mathrm{L}$ were injected into a loop, $10 \mu \mathrm{L}$ of which were transferred to the column. All samples were measured in triplicate.

\section{Results and Discussion}

Commercial $100 \mathrm{mg}$ Claritin tablets are composed of $10 \mathrm{mg}$ loratadine (the antihistamine API), and magnesium stearate, lactose monohydrate, and corn starch at un-labelled mass amounts [24]. The Raman measurement parameters, i.e., laser power, integration time, and spectral averages, were examined to optimize the measurement of loratadine and the Claritin excipients. It was found that $275 \mathrm{~mW}$ at $1064 \mathrm{~nm}, 10$ averaged, 2-s integration scans produced quality spectra. In the case of the loratadine, magnesium stearate, lactose monohydrate, and corn starch reference tablets, 100 spectra were averaged to yield high signal-to-noise ratio spectra. These spectra subsequently served as the reference spectra for tablet analysis using the S-Quant software. The Raman spectra for loratadine and magnesium stearate are clearly different than those of lactose monohydrate, and corn starch, however the latter two spectra share several common vibrational modes, as their chemical structures suggest (Figure 1). 


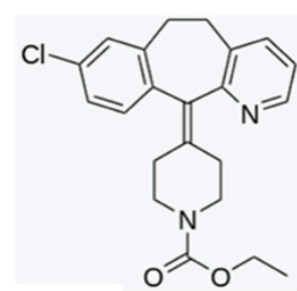

Loratadine

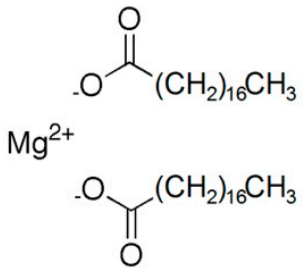

Magnesium Stearate

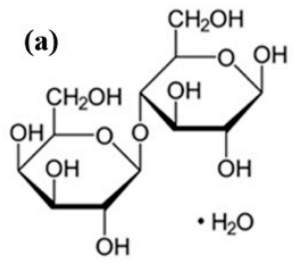

Lactose Monohydrate

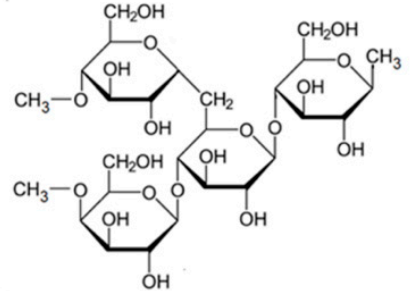

Cornstarch
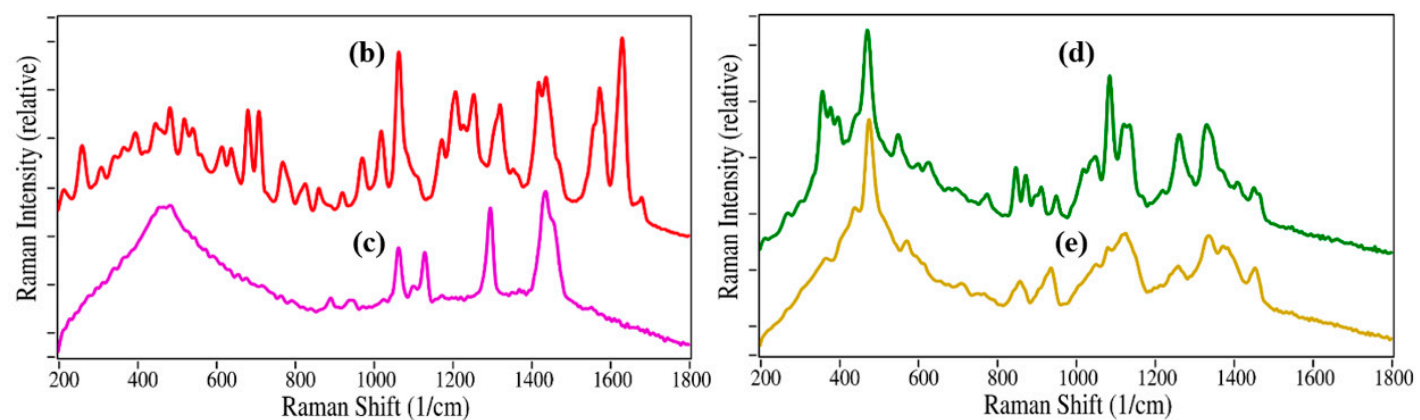

Figure 1. (a) Chemical structures for all four Claritin chemicals. Raman spectra of (b) loratadine, (c) magnesium stearate, (d) lactose monohydrate, and (e) cornstarch. Sample conditions: Tablets (pressed at 1500 psi). Spectral Conditions: $275 \mathrm{~mW}$ at $1064 \mathrm{~nm}, 10$ averaged, 2-s integration scans/spectrum (total time was $200 \mathrm{~s}$ ).

An initial Claritin tablet was used to examine the uniform distribution of loratadine in the tablet. This was accomplished by measuring the Raman spectra of 81 discrete points in a $9 \times 9$ point grid covering a $4 \times 4 \mathrm{~mm}^{2}$ to a depth of $\sim 1 \mathrm{~mm}$ section of the tablet. The loratadine mass percent was calculated using two methods. First, the raw spectra were analyzed by simply measuring the baseline corrected $1630 \mathrm{~cm}^{-1}$ carbonyl peak height. The height for each tablet was divided by the average height from all the tablets multiplied by 10, the expected mass percentage. Second, the 1st-derivative, smoothed spectra from 200 to $1800 \mathrm{~cm}^{-1}$ were fit with weighted percentages of pure loratadine, magnesium stearate, corn starch and lactose monohydrate, the total of which was set to $100 \%$ (Figure S1a). The 1st-derivative was used to remove the effects of baseline offset and slope. The software indicated that magnesium stearate ranged from -2 to $+2 \%$, which was not surprising, as this ingredient is used as a mold release agent typically at $\sim 1 \%$ [25]. Consequently, magnesium stearate was excluded from further analysis. Not unexpectedly, the peak height method yielded somewhat more scatter in the loratadine mass percent than the full spectrum fitting method. The former ranged from 8.0 to 11.6 mass percent, while the latter ranged from 9.2 to 11.5 mass percent, both indicating uniform distribution of this API throughout the tablet. To further illustrate the API distribution, the loratadine mass percentages for both methods were plotted as $4 \mathrm{~mm} \times 4 \mathrm{~mm}$ "heat maps", in which red was set to 12.0 mass percent and blue to 8.0 mass percent (Figure 2). Overall, these high color-contrast maps show similar distribution of the loratadine, with the highest mass percent in the upper left corner, and lower mass percents in the middle. It is worth noting that the spectral fit method yielded lactose monohydrate and cornstarch mass percentages of 74.4 and $15.6 \%$ for the $100 \mathrm{mg}$ tablet. While the excipient mass percentages are not given on the product label, they are likely intended to be 75 and 15 mass percent, since the Summary of Product Characteristics for a generic loratadine product sold by Actavis UK Ltd. (Devon, UK) lists the former as $75 \mathrm{mg}$ per $100 \mathrm{mg}$ tablet [26]. 


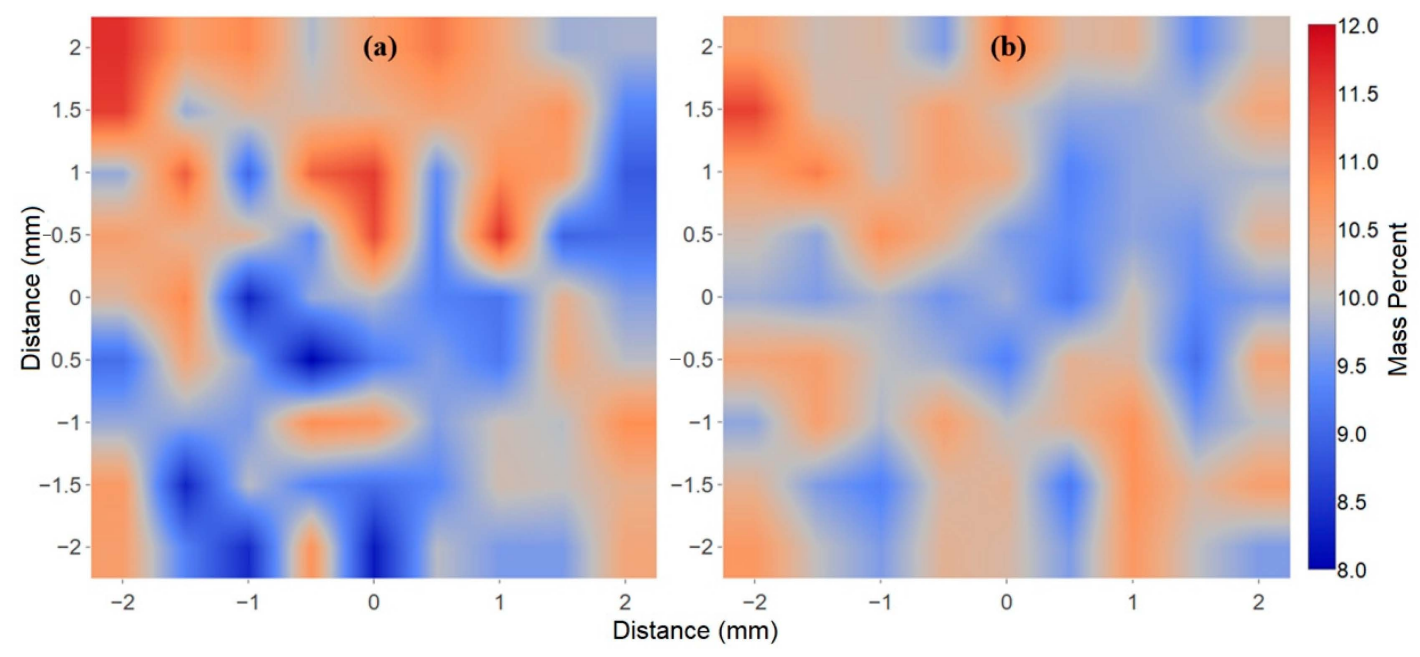

Figure 2. False color heat maps showing the loratadine mass percent (blue $=8 \%$, red $=12 \%$ ) for a $9 \times 9$ point grid covering a $4 \times 4 \mathrm{~mm}^{2}$ surface section to a depth of $\sim 1 \mathrm{~mm}$ of a Claritin tablet using 81 Raman spectra determined by (a) the $1630 \mathrm{~cm}^{-1}$ peak height (baseline corrected) and (b) the weighted percentage, 1st-derivative, $200-1800 \mathrm{~cm}^{-1}$, spectral fits using pure loratadine, lactose monohydrate and cornstarch. Spectral conditions: $275 \mathrm{~mW}$ at $1064 \mathrm{~nm}, 10$ averaged, 4-s integrations/spectrum (total time at $40 \mathrm{~s} / \mathrm{spot}$ was $\sim 1 \mathrm{~h}$ ).

Since it is critical to provide the public with medications that contain the label specified API dosage, the US Pharmacopeia developed a method for pharmaceutical companies to calculate an acceptance value, $\mathrm{AV}$, which indicates the API content uniformity of a production batch and if it passes, i.e., can be released to the public [21]. The AV is calculated in terms of the mean of the API contents, Xbar, expressed as a percentage of the label claim, $\mathrm{k}$, an acceptance constant ( $\mathrm{k}=2.4$ for 10 samples, $\mathrm{k}=2.0$ for 30 samples), and the sample standard deviation, $\mathrm{s}$, for three conditional cases based on the value of the mean, Xbar, according to the following Equations (1)-(3):

$$
\begin{gathered}
\mathrm{AV}=\mathrm{ks}, \text { if } 98.5 \% \leq \mathrm{Xbar} \leq 101.5 \%, \\
\mathrm{AV}=98.5-\mathrm{Xbar}+\mathrm{ks}, \text { if } \mathrm{Xbar}<98.5 \%, \\
\mathrm{AV}=\mathrm{Xbar}-101.5+\mathrm{ks}, \text { if } \mathrm{Xbar}>101.5 \% .
\end{gathered}
$$

In general, 10 samples from a batch are analyzed to determine if the API is within $15 \%$ of the label claimed dosage. If the calculated $\mathrm{AV}$ is modestly greater than $15 \%$, then an additional 20 samples are analyzed. This procedure was followed first for 10, then 30 total Claritin tablets. As described above, each tablet was measured for $\sim 3$ min (200 s) using $275 \mathrm{~mW}$ at $1064 \mathrm{~nm}$. The loratadine mass percentage for each tablet was calculated by fitting 1st-derivative Raman spectrum of each tablet with the pure spectra of the loratadine, lactose monohydrate, and cornstarch 1500 psi tablets using the S-Quant software (Figure S1a). It was found that the first 10 tablets yielded a mean value of 9.72 mass percent per tablet or $97.2 \%$ of the loratadine label claim. Using Equation (2), an AV of $11.9 \%$ was obtained, which, being less than $15 \%$, indicates the batch passes. The same procedure was followed for all 30 tablets, yielding $97.0 \%$ of the loratadine label claim, and an $\mathrm{AV}$ of $9.3 \%$, again passing the batch (Table 1 and Table S1). Even though only 9 points were measured per tablet, it appears to be a good compromise in terms of determining the fate of the batch and the time required to make the measurement. It is worth noting that calculating the loratadine mass percentage for the same 30 tablets using the tablet-to-pure 1500 psi loratadine tablet ratio of the $1630 \mathrm{~cm}^{-1}$ Raman peak heights, baseline offset and tilt corrected, yielded $7.75 \mathrm{mg}$ per tablet, with an AV of 31.6, indicating that the batch should be rejected (Table 1, note that the calculated 10-tablet AV using peak heights was worse). 
Table 1. Calculated Acceptance Values for 10 and 30 tablets each of Claritin and a generic product based on loratadine mass percents by HPLC, Raman chemical component analysis, and Raman created composite excipient analysis.

\begin{tabular}{|c|c|c|c|c|c|c|c|}
\hline Loratadine & $\begin{array}{l}\text { Number of } \\
\text { Samples, } n\end{array}$ & Mean, X-bar & $\begin{array}{l}\text { Acceptance } \\
\text { Constant, k }\end{array}$ & $\begin{array}{c}\text { Standard } \\
\text { Deviation, s }\end{array}$ & EquationUsed & $\begin{array}{l}\text { Acceptance } \\
\text { Value, AV }\end{array}$ & $\begin{array}{c}\text { Pass? } \\
(<15 \% ?)\end{array}$ \\
\hline \multicolumn{8}{|l|}{ Claritin } \\
\hline $\begin{array}{c}\text { Raman Pure } \\
\text { Chemicals }\end{array}$ & 10 & 97.2 & 2.4 & 4.4 & 2 & 11.9 & Yes \\
\hline $\begin{array}{l}\text { Raman Pure } \\
\text { Chemicals }\end{array}$ & 30 & 97.0 & 2.0 & 3.9 & 2 & 9.3 & Yes \\
\hline $\begin{array}{c}\text { Raman } 1630 \mathrm{~cm}^{-1} \\
\text { Peak Height }\end{array}$ & 10 & 77.3 & 2.4 & 6.8 & 2 & 37.6 & No \\
\hline $\begin{array}{l}\text { Raman } 1630 \mathrm{~cm}^{-1} \\
\text { Peak Height }\end{array}$ & 30 & 77.5 & 2.0 & 5.3 & 2 & 31.6 & No \\
\hline HPLC & 10 & 98.7 & 2.4 & 5.6 & 1 & 13.5 & Yes \\
\hline HPLC & 30 & 102.1 & 2.0 & 6.3 & 3 & 13.2 & Yes \\
\hline $\begin{array}{c}\text { Raman Created } \\
\text { Excipient }\end{array}$ & 10 & 97.8 & 2.4 & 4.1 & 2 & 10.6 & Yes \\
\hline $\begin{array}{c}\text { Raman Created } \\
\text { Excipient }\end{array}$ & 30 & 97.9 & 2.0 & 4.0 & 2 & 8.7 & Yes \\
\hline \multicolumn{8}{|l|}{ Generic } \\
\hline $\begin{array}{c}\text { Raman Created } \\
\text { Excipient }\end{array}$ & 10 & 102.5 & 2.4 & 3.9 & 3 & 10.3 & Yes \\
\hline $\begin{array}{c}\text { Raman Created } \\
\text { Excipient }\end{array}$ & 30 & 101.2 & 2.0 & 4.0 & 1 & 8.0 & Yes \\
\hline
\end{tabular}

The same 30 tablets were then individually analyzed by high-performance liquid chromatography, the standard method for determining acceptance values for drugs [27-29]. Since these measurements employed manual sample introduction into the injection loop, all samples were prepared with $0.1 \mathrm{mg} / \mathrm{mL}$ desloratadine as an internal concentration standard. The standard was measured 10 times on the two days that HPLC was performed, and the averaged integrated peak areas were used to correct the loratadine peak areas. Prior to analysis, a calibration curve was developed by measuring loratadine concentrations ranging from 0.05 to $0.25 \mathrm{mg} / \mathrm{mL}$. The loratadine concentration standards, measured in triplicate, produced excellent concentration-to-peak area linearity over the desired concentration range (Figure 3a). For each sample, three $10-\mu \mathrm{L}$ injections, which produced desloratadine and loratadine peaks with retention times at 1.26 and $2.77 \mathrm{~min}$ with peak heights of $\sim 7 \times 10^{-5}$ and $4.5 \times 10^{-5}$ absorbance units, respectively, were averaged to calculate their concentrations. The values were also corrected by the measured mass of each tablet.

As before, the acceptance values were calculated for the first 10 tablets, then all 30 tablets. Analysis of the first 10 tablets yielded a mean value of $9.87 \mathrm{mg}$ per tablet or $98.7 \%$ of the loratadine label amount. Therefore, Equation (1) was used (Table 1). An AV of $13.2 \%$ was obtained, less than $15 \%$, passing the batch. In the case of all 30 tablets, the mean percent of the label amount was $101.6 \%$, so Equation (3) was used, which yielded an AV of $14.1 \%$, just below $15 \%$, but still passing the batch. 
(a)

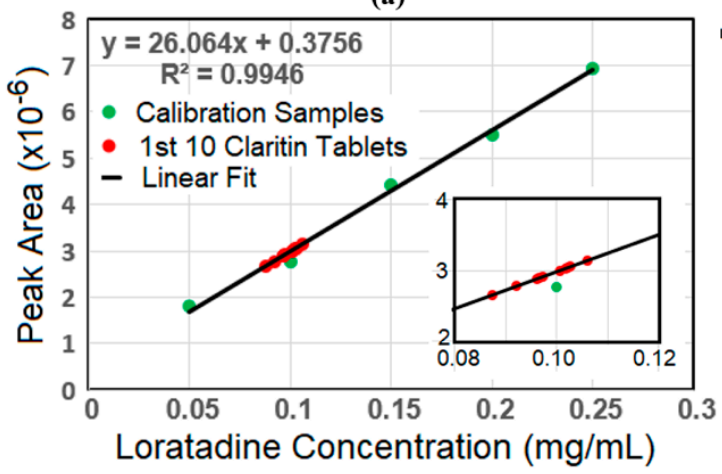

(b)

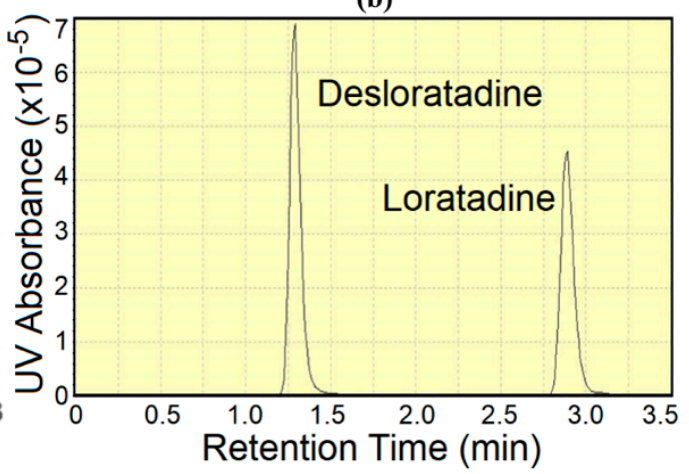

Figure 3. (a) HPLC-generated concentration curve for loratadine with calculated concentrations for the 1st 10 Claritin tablets (inset-expanded view of 10 tablets). (b) Example chromatograph with desloratadine and Claritin tablet elution peaks. Desloratadine and loratadine peak areas are 2,747,919 and 2,483,413, respectively.

As indicated by these analyses, both methods gave mean tablet loratadine mass percentages close to $10 \%$ of the total mass, or $10 \mathrm{mg}$ for the $100 \mathrm{mg}$ tablets, and both methods passed the batch based on measuring 10 and 30 tablets. However, the percentage coefficient of variation for the HPLC 30 tablet data was significantly greater than the Raman 30 tablet data using the created excipient spectrum, viz: $6.2 \%$ versus $4.1 \%$ (Table S1). It is also interesting to note that tablet 12 produced the highest mass percent by both techniques, 11.0 and 10.7 mass percent for HPLC and Raman, respectively. However, there was no such correspondence for the lowest values; Tablet 3 at 8.8 mass percent for HPLC and Tablet 11 at 9.1 mass percent for Raman and (Figure $4 a$ ).

(a)

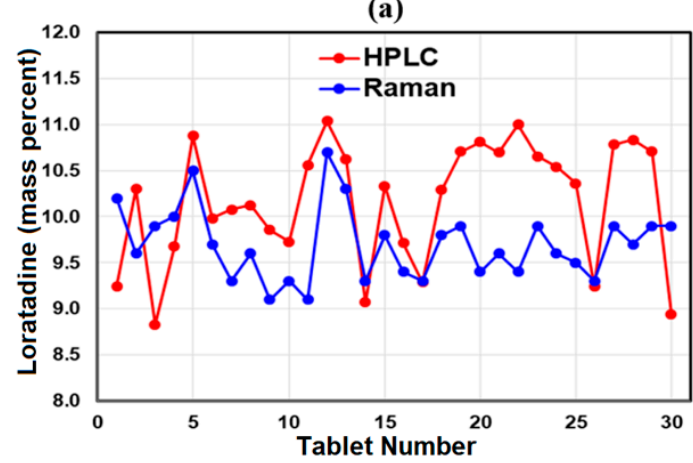

(b)

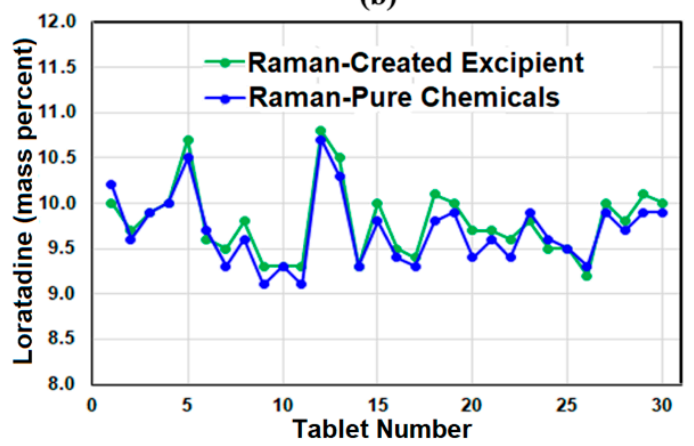

Figure 4. Plots of loratadine mass percentage as a function of tablet number for (a) HPLC and Raman using all excipients, and (b) Raman using all excipients and a created composite excipient.

While the above data suggest that Raman spectroscopy is as good as, or better than, HPLC for determining API mass percents to qualify batches, it required the Raman spectra of the excipients, which may be difficult to obtain or are unknown. These limitations can be overcome by creating a composite excipient spectrum by simply subtracting the spectrum of pure loratadine from the 10 and 30 tablet averaged spectra, respectively (Figure 5a). For this case, the S-Quant software was used to determine the loratadine mass percent for the 10 and 30 tablets using weighted percentages of the pure loratadine 1st-derivative Raman spectrum and the corresponding composite excipient spectrum (Figure S1b). 

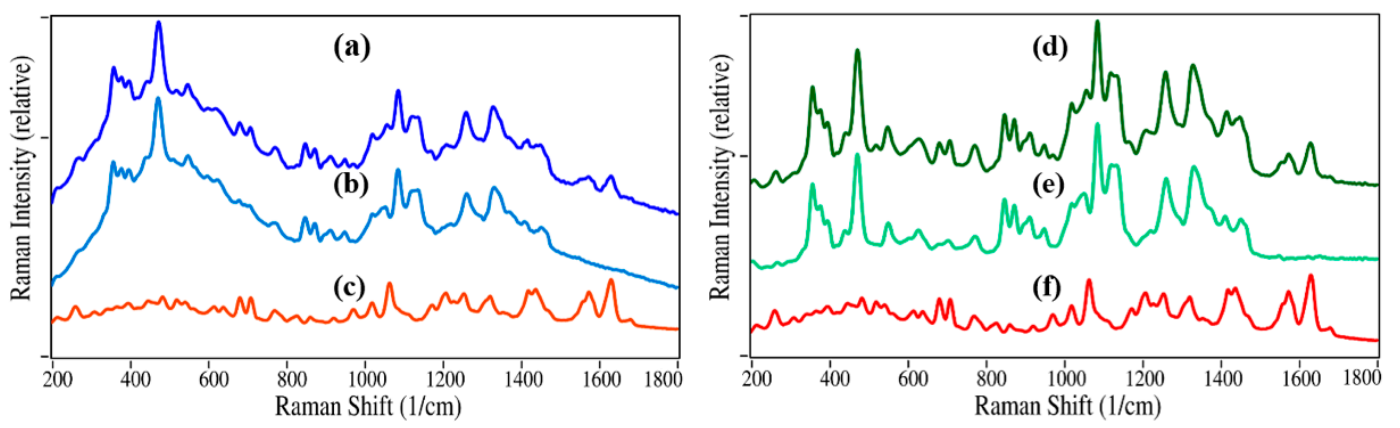

Figure 5. Raman spectra of (a) Claritin (30 tablet average), (b) the excipient created by subtracting (c) loratadine from (a,d) a generic for Claritin (30 tablet average), (e) the excipient created by subtracting (f) loratadine from (d). Spectral Conditions: $275 \mathrm{~mW}$ at $1064 \mathrm{~nm}, 10$ averaged, 2-s integration scans. Spectra are offset for clarity. Intensities are the same scale, except loratadine spectra (c) and (f), which were increased by a factor of 2 .

Again, the acceptance values were calculated for the first 10 tablets, then all 30 tablets. Analysis of the first 10 and 30 tablets yielded mean percentages of 97.8 and $97.9 \%$ of the loratadine label amount, respectively. Since these values were both less than $98.5 \%$, Equation (2) was used to calculate their respective AVs, which were 10.6 and $8.7 \%$, respectively, both less than $15 \%$, passing the batches. The created excipient spectrum AV values are slightly better than those obtained using lactose monohydrate and cornstarch pure chemical spectra (Table 1). This improvement is attributed to using one less variable, i.e., one created excipient spectrum instead of two chemical spectra. It is also worth noting that the individual calculated mass percentages for the 30 tablets are nearly identical, with a percentage coefficient of variation only $0.07 \%$ higher than the lactose monohydrate and cornstarch-based mass percentages (Figure $4 b$, Table S1).

The use of a created excipient to determine the API mass percentage for a drug product was taken one step further. As stated in the introduction, many drugs use multiple excipients, and their relative mass percentages may be a trade secret. While this may not be a matter for the original manufacturer, it may be important in forensic studies or identifying counterfeit drugs. To demonstrate this approach, the Raman spectra for a batch of thirty $10 \mathrm{mg}$ per $100 \mathrm{mg}$ loratadine product tablets, as a generic for Claritin, were measured. Then, the pure loratadine spectrum was subtracted from first 10 then 30 tablet averaged spectra to create excipient spectra (Figure 5). The excipient spectra for the generic are considerable different than the Claritin-generated excipient spectra (compare Figure $5 b$ to Figure $5 e$ ).

Once again, the acceptance values were calculated for the first 10 tablets, then all 30 tablets. Analysis of the first 10 and 30 tablets yielded mean percentages of $102.5 \%$ and $101.2 \%$ of the loratadine label amount, respectively. The AVs were then calculated according to Equations (3) and (1), to yield 10.3 and $8.0 \%$, respectively, both less than $15 \%$, and both batches passed (Table 1 and Table S1). These values are slightly better than those obtained using lactose monohydrate and cornstarch spectra.

It is worth noting that the Raman-based created excipient method required less than $5 \mathrm{hr}$ : $\sim 1 \mathrm{hr}$ to prepare the $1500 \mathrm{psi}$ loratadine tablet, $\sim 1.5 \mathrm{hr}$ to measure the loratadine and 30 Claritin tablets at $3 \times 3$ points each, $\sim 1 \mathrm{hr}$ to fit the spectra and determine the loratadine mass percent for each tablet, and $\sim 1 \mathrm{hr}$ for the USP analysis. In contrast, the HPLC method required more than $13 \mathrm{hr}: \sim 1 \mathrm{hr}$ to prepare buffer solutions and carrier solvents, $\sim 1 \mathrm{~h}$ to prepare the reference solution and the calibration standards, $\sim 2 \mathrm{hr}$ to weigh and prepare the 30 individual sample solutions, $\sim 2 \mathrm{hr}$ to pre-treat the column, $\sim 4 \mathrm{hr}$ to measure the 15 calibration and 30 Claritin samples, $\sim 2 \mathrm{hr}$ to determine the loratadine mass percent for each tablet, and $\sim 1 \mathrm{hr}$ for the USP analysis. In addition to analysis time, the development of the Raman method for a new drug only requires preparing and measuring a pressed tablet of the target API, and establishing the best spectral region to use, whereas, the development of an HPLC method requires selecting a column, appropriate buffers, solvents, a reference material, and the measurement conditions. The former can be accomplished in 1 day, while 
they latter typically takes a week or more [29]. It is of course realized that the Raman method relies on uniform distribution of the API in the tablet, and may be limited to relatively thin tablets, such as those less than $3 \mathrm{~mm}$ thick. The analyses of the Claritin and generic tablets clearly indicate that such uniformity exists in these tablets, and likely in similar products.

\section{Conclusions}

Here, we demonstrated a method to determine the API mass percent of a drug product without knowing the excipients, by subtracting the API Raman spectrum from the product Raman spectrum to create a composite excipient spectrum that could be used in mass percentage calculations. The method proved equivalent to the standard HPLC method, at least for Claritin tablets. We believe that the method could be used to (1) qualify content uniformity of a manufactured drug product batch in accordance with the prescribed USP method, even if the excipients are unknown; and (2) identify counterfeit drugs without needing to know the identity or concentration of excipients of the purported drug product.

Supplementary Materials: The following are available online at https:/ /www.mdpi.com/1999-492 3/13/3/309/s1, Figure S1: Images of S-quant software used to fit the 1st-derivative Raman spectrum of (a) a single Claritin tablet with loratadine, corn starch, and lactose monohydrate 1st-derivative Raman spectra, and (b) the 1st-derivative Raman spectrum of a single generic tablet with loratadine and a created excipient 1st-derivative Raman spectra. Measured Raman spectra are shown for clearer presentation, Table S1: Calculated loratadine mass percents with statistics for 1st 10 and all 30 Claritin and a generic tablets by HPLC, Raman chemical component analysis, Raman created excipient analysis, and $1630 \mathrm{~cm}^{-1}$ peak height analysis.

Author Contributions: Conceptualization, S.F., C.S. and C.B.; methodology, A.F., Z.G. and J.C.; software, W.S. and A.F.; validation, A.F., S.F.; formal analysis, A.F., S.F. and G.R.; resources, C.B.; data curation, A.F.; writing—original draft preparation, S.F.; writing—review and editing, A.F.; funding acquisition, S.F. All authors have read and agreed to the published version of the manuscript.

Funding: The authors are grateful for support from NASA's Johnson Space Center, Ames Research Center, and SBIR program (NNX14C08C and NNX16CA16C).

Institutional Review Board Statement: Not applicable.

Informed Consent Statement: Not applicable.

Data Availability Statement: Not applicable.

Acknowledgments: We thank Kathryn Dana and Evan Cole for modifying the stage positioning software and preparing some of the HPLC samples, respectively.

Conflicts of Interest: The funders had no role in the design of the study; in the collection, analyses, or interpretation of data; in the writing of the manuscript, or in the decision to publish the results. All authrors worked at RTA at the time the work was performed, except, Gary Ritchie, who was a consultant. Amelia is a now a Senior studying Areospace Engineering at RPI.

\section{References}

1. Cutmore, E.A.; Skett, P.W. Application of Fourier-transform Raman spectroscopy to a range of compounds of pharmaceutical interest. Spectrochim. Acta 1993, 49A, 809-818. [CrossRef]

2. Petty, C.J.; Bugay, D.E.; Findlay, W.P.; Rodreigez, C. Applications of FT-Raman spectroscopy in the pharmaceutical industry. Spectroscopy 1996, 11, 41-45.

3. Hendra, P.J. Fourier-transform Raman spectroscopy in pharmaceutical analysis and research. Am. Lab. 1996, 28, 17-24.

4. Frank, C. Review of pharmaceutical applications of Raman spectroscopy. In Analytical Applications of Raman Spectroscopy; Pellitier, M.J., Ed.; Blackwell Science: Oxford, UK, 1999; pp. 224-271.

5. Vergote, G.J.; De Beer, T.R.M.; Vervaet, C.; Remon, J.P.; Baeyens, W.; Diericx, N.; Verpoort, F. In-Line Monitoring of a Pharmaceutical Blending Process Using FT-Raman Spectroscopy. Eur. J. Pharm. Sci. 2004, 21, 479-485. [CrossRef]

6. Sasic, S.; Clark, D.A. Defining a strategy for chemical imaging of industrial pharmaceutical samples on Raman line-mapping and global illumination instruments. Appl. Spectrosc. 2006, 60, 494-502. [CrossRef] [PubMed]

7. Wikstrom, H.; Romero-Torres, S.; Wongweragiat, S.; Williams, J.A.S.; Grant, E.R.; Taylor, L.S. On-Line Content Uniformity Determination of Tablets Using Low-Resolution Raman Spectroscopy. Appl. Spectrosc. 2006, 60, 672-681. [CrossRef] [PubMed] 
8. Kim, J.; Noh, J.; Chung, H.; Woo, Y.A.; Kemper, M.S.; Lee, Y. Direct, non-destructive quantitative measurement of an active pharmaceutical ingredient in an intact capsule formulation using Raman spectroscopy. Anal. Chim. Acta 2007, 598, $280-285$. [CrossRef]

9. Roggo, Y.; Degardin, K.; Margot, P. Identification of pharmaceutical tablets by Raman spectroscopy and chemometrics. Talanta 2010, 81, 988-995. [CrossRef] [PubMed]

10. Sacré, P.Y.; Deconinck, E.; Saerens, L.; De Beer, T.; Courselle, P.; Vancauwenberghe, R.; Chiap, P.; Crommen, J.; De Beer, J.O. Detection of counterfeit Viagra ${ }^{\circledR}$ by Raman microspectroscopy imaging and multivariate analysis. J. Pharm. Biomed. Anal. 2011, 56, 454-461. [CrossRef]

11. Lanzarotta, A.; Lorenz, L.; JaCinta, S.; Flurer, C. Development and implementation of a pass/fail field-friendly method for detecting sildenafil in suspect pharmaceutical tablets using a handheld Raman spectrometer and silver colloids. J. Pharm. Biomed. Anal. 2017, 146, 420-425. [CrossRef] [PubMed]

12. Farquharson, S. Pharmaceutical applications of Raman spectroscopy. Am. Pharm. Rev. 2014, 17, 32-39.

13. Breitenbach, J.; Schrof, W.; Neumann, J. Confocal Raman spectroscopy: Analytical approach to solid dispersions and mapping of drugs. Pharm. Res. 1999, 16, 1109-1113. [CrossRef]

14. Schlucker, S.; Schaeberle, M.D.; Huffman, S.W.; Levin, I.W. Raman Microspectroscopy: A Comparison of point, line, and wide-field imaging methodologies. Anal. Chem. 2003, 75, 4312-4318. [CrossRef] [PubMed]

15. El Hagrasy, A.S.; Chang, S.-Y.; Desai, D.; Kiang, S. Raman spectroscopy for the determination of coating uniformity of tablets: Assessment of product quality and coating pan mixing efficiency during scale-up. J. Pharm. Innov. 2006, 1, 37-42. [CrossRef]

16. Matousek, P.; Parker, A.W. Non-invasive probing of pharmaceutical capsules using transmission Raman spectroscopy. J. Raman Spectrosc. 2007, 38, 563. [CrossRef]

17. Adar, F.; Lee, E.; Whitley, A. Transmission Raman Offers Improved Quantitation of Pharmaceutical Solids. Available online: https: //www.spectroscopyonline.com/view/transmission-raman-offers-improved-quantitation-pharmaceutical-solids (accessed on 1 February 2021).

18. Porto, S.L.S.; Wood, D.L. Ruby optical maser as a Raman source. J. Opt. Soc. Am. 1962, 52, 139-141. [CrossRef]

19. Townsend, N.; Nordon, A.; Littlejohn, D.; Myrick, M.; Andrews, J.; Dallin, P. Comparison of the determination of a lowconcentration active ingredient in pharmaceutical tablets by backscatter and transmission Raman spectrometry. Anal. Chem. 2012, 84, 4671-4676. [CrossRef] [PubMed]

20. Schering-Plough Corporation, Global Safety \& Environmental Affairs, which States "These Products are Exempt from Material Safety Data Sheet Requirements: .. Claritin D-24." MSDS SP001447, a Similar Product to Claritin, Reditabs States "The Formulation for This Product Is Proprietary Information." 2010. Available online: https:/ /imgcdn.mckesson.com/CumulusWeb/Click_ and_learn/MSDS_9MSDCC_04110080554_CLARITIN_REDITABS_FOR_KIDS_12-HOUR.pdf (accessed on 1 February 2021).

21. Uniformity of Dosage Units. Proceedings of the United States Pharmacopeial Convention; United States Pharmacopoeia: Rockville, MD, USA, 2017; pp. 736-740.

22. Shende, C.; Smith, W.; Brouillette, C.; Farquharson, S. Drug Stability Analysis by Raman Spectroscopy. Pharmaceutics 2014, 6, 651-662. [CrossRef] [PubMed]

23. Loratadine Oral Solution; Revision Bulletin; United States Pharmacopeia: Rockville, MD, USA, 1 May 2011.

24. Bayer, PLC. Summary of Product Characteristics "Claritin Allergy 10 mg Tablets". Available online: https://www.medicines.org. uk/emc/product/3505/smpc\#gref (accessed on 1 February 2021).

25. Shadangi, M.; Seth, S.; Senapati, D. Critical roles of magnesium stearate in formulation development of a highly soluble drug metformin hyrochloride. Int. J. Pharm. Sci. Res. 2012, 3, 1188-1193.

26. Actavis UK Ltd. Summary of Product Characteristics "Loratadine 10 mg Tablets". This Company Has Been Acquired. Available online: https: / / www.medicines.org.uk/emc/product/4501/smpc\#gref (accessed on 1 February 2021).

27. Ruperez, F.J.; Fernandez, H.; Barbas, C. LC determination of loratadine and related impurities. J. Pharm. Biomed. Anal. 2002, 29, 35-41. [CrossRef]

28. El-Sherbiny, D.T.; El-Enany, N.; Belal, F.F.; Hansen, S.H. Simultaneous determination of loratadine and desloratadine in pharmaceutical preparations using liquid chromatography with a microemulsion as eluent. J. Pharm. Biomed. Anal. 2007, 43, 1236-1242. [CrossRef] [PubMed]

29. Pavalache, G.; Dorneanu, V.; Popescu, A. Validation and application of a new DAD-HPLC method for determination of loratadine from pharmaceuticals. Farmacia 2015, 63, 366-370. 